\title{
Mutational analysis of the Kir6.1 gene in Chinese hypertensive patients treated with the novel ATP-sensitive potassium channel opener iptakalim
}

\author{
RUIFENG DUAN $^{1}$, WENYU CUI $^{1}$ and HAI WANG ${ }^{1,2}$ \\ ${ }^{1}$ Cardiovascular Drug Research Center, Institute of Health and Environmental Medicine, Academy of Military \\ Medical Sciences, Beijing 100850; ${ }^{2}$ Thadweik Academy of Medicine, Beijing 100039, P.R. China
}

Received March 8, 2011; Accepted April 27, 2011

DOI: $10.3892 /$ etm.2011.259

\begin{abstract}
Iptakalim is a novel $\mathrm{K}_{\mathrm{ATP}}$ opener with antihypertensive properties. The Kir6.1 gene is one of the candidate genes that may influence the response to iptakalim in hypertensive patients. We aimed to ascertain whether a mutation in the coding region of the Kir6.1 gene is present in Chinese Han hypertensive patients. The study population included 162 Chinese Han hypertensive patients (81 men and 81 women with a mean age of $55 \pm 9$ years). Mutational analysis of the coding region of Kir6.1 was performed using PCR-SSCP and direct sequencing. No missense or nonsense mutations were found in these samples, while a single base pair substitution ( $\mathrm{C}$ to $\mathrm{T}$ ) at nucleotide position 111 in exon 2 of the coding region was noted in four patients (one female and three male), who were all heterozygous for the mutation. This $\mathrm{C}$ to $\mathrm{T}$ substitution did not result in an amino acid substitution (Ile37Ile, silent mutation). In the remaining 158 patients, no mutation was detected. The blood pressure of these four patients carrying the I37I polymorphism was well controlled by iptakalim. No mutation that alters the primary structure of Kir6.1 was detected in Chinese Han hypertensive patients. The results indicate that abnormality in the primary structure of Kir6.1 is not involved in the genetic pathogenesis of essential hypertension in Chinese Han hypertensive patients and has no effect on the BP response to iptakalim treatment.
\end{abstract}

\section{Introduction}

Essential hypertension is the leading cause of cardiovascular morbidity and mortality worldwide, affecting $\sim 20 \%$ of the adult population (1). Many drugs are effective in treating

Correspondence to: Dr Hai Wang, Cardiovascular Drug Research Center, Institute of Health and Environmental Medicine, Academy of Military Medical Sciences, Beijing 100850, P.R. China

E-mail:wh9588@yahoo.com.cn

Key words: hypertension, Kir6.1 gene, mutation, iptakalim hypertension, although individuals can respond differently to the same drug. Interindividual variation in the efficacy of medications may be influenced by genetic variations (2). Hypertension pharmacogenetics seeks to find genetic predictors of response to drugs that lower blood pressure. More and more studies have investigated associations between genetic polymorphisms and response to drugs (3-6).

$\mathrm{K}_{\text {ATP }}$ (ATP-sensitive potassium channel) channels have important functions through their coupling of cellular energetic networks and their ability to decode metabolic signals, and they are implicated in diseases of many organs. $\mathrm{K}_{\mathrm{ATP}}$ is formed by the physical association between the inwardrectifier potassium channels (Kir6.x) and the regulatory sulfonylurea receptor subunit (SUR), which forms a heterooctameric complex (7). Iptakalim is a novel $\mathrm{K}_{\mathrm{ATP}}$ opener with antihypertensive properties targeting small arteries of hypertensive status in different models of hypertension in rats and dogs. Iptakalim can produce long-lasting hypotensive effects without tolerance. At the same time it exerted a protective effect against hypertensive damage to target organs. Iptakalim has preferentially selective effects on SUR2B/Kir6.1 and it is a more potent activator of the SUR2B/Kir6.1 subtype of $\mathrm{K}_{\mathrm{ATP}}$ channels than diazoxide and pinacidil, the two most commonly studied $\mathrm{K}_{\text {ATP }}$ channel openers (8-14). Iptakalim is an effective antihypertensive drug for the treatment of mild to moderate essential hypertension (15). Yet, individual variations for its antihypertensive effects have been observed. Genetic variations that alter the structure, configuration, activity, or quantity of the drug target receptors or target-related regulation factors may contribute to individual variations in drug response (16). Thus, genetic variations of the Kir6.1 gene may contribute to individual variations in drug response.

In the present study, we investigated polymorphisms of the Kir6.1 gene in 162 Chinese Han hypertensive patients and their possible association with the antihypertensive response to iptakalim treatment.

\section{Materials and methods}

Patients. All of the material studied was obtained from subjects who completed the protocol at 5 centers of the phase III clinical trial of iptakalim which was a randomized double-blind 
Table I. Primers and parameters for the PCR procedure used in this study.

\begin{tabular}{|c|c|c|c|}
\hline Fragment & & Sequence & Amplicon size (bp) \\
\hline $\mathrm{F} 1$ & $\begin{array}{l}\text { Sense } \\
\text { Antisense }\end{array}$ & $\begin{array}{l}\text { 5'-GGTGAGGATAGGGTGGGTTT-3' } \\
\text { 5'-CCTGTAGAAAGCGTCCTTGC-3' }\end{array}$ & 297 \\
\hline $\mathrm{F} 2$ & $\begin{array}{l}\text { Sense } \\
\text { Antisense }\end{array}$ & $\begin{array}{l}\text { 5'-ACCTGGCGCATAAGAACATC-3' } \\
\text { 5'-CACACAGTGGACTCCAAACC-3' }\end{array}$ & 229 \\
\hline F3 & $\begin{array}{l}\text { Sense } \\
\text { Antisense }\end{array}$ & $\begin{array}{l}\text { 5'-CTATCATGTGGTGGCTGGTG-3' } \\
\text { 5'-TGTTAGGTGTTGTTCTTTATTGTGC-3' }\end{array}$ & 272 \\
\hline $\mathrm{F} 4$ & $\begin{array}{l}\text { Sense } \\
\text { Antisense }\end{array}$ & $\begin{array}{l}\text { 5'-TCAAAATGTGACCTGATGGTG-3' } \\
\text { 5'-GTTTCTGCCCTTCTGTGAGC-3' }\end{array}$ & 242 \\
\hline F5 & $\begin{array}{l}\text { Sense } \\
\text { Antisense }\end{array}$ & $\begin{array}{l}\text { 5'-GCCATCACGGTTTTGATTCT-3' } \\
\text { 5'-CAGGAATGTCCAGTTGGTGA-3' }\end{array}$ & 286 \\
\hline F6 & $\begin{array}{l}\text { Sense } \\
\text { Antisense }\end{array}$ & $\begin{array}{l}\text { 5'-GAGTGGGTGACCTGAGGAAA-3' } \\
\text { 5'-GCCAGGTCAGTTGCTGAGAT-3' }\end{array}$ & 226 \\
\hline F7 & $\begin{array}{l}\text { Sense } \\
\text { Antisense }\end{array}$ & $\begin{array}{l}\text { 5'-GATCATCTGCCACGTGATTG-3' } \\
\text { 5'-GGTTTCTCATCCAGCTCTCG-3' }\end{array}$ & 291 \\
\hline F8 & $\begin{array}{l}\text { Sense } \\
\text { Antisense }\end{array}$ & $\begin{array}{l}\text { 5'-ATCACCACACAAGCACGAAC-3' } \\
\text { 5'-TGCGCTTCCTCAGAGAATTT-3' }\end{array}$ & 238 \\
\hline F9 & $\begin{array}{l}\text { Sense } \\
\text { Antisense }\end{array}$ & $\begin{array}{l}\text { 5'-CGAGAGCTGGATGAGAAACC-3' } \\
\text { 5'-CCCAGCATAAACCGTCAAAA-3' }\end{array}$ & 264 \\
\hline
\end{tabular}

trial performed at 14 centers in 11 cities in China. We studied 162 non-related Chinese Han patients with essential hypertension ( 81 men, 81 women) (mean age $\pm \mathrm{SD}, 55 \pm 9$ years; range, 26-74). Patients met the World Health OrganizationInternational Society of Hypertension criteria for hypertension [systolic BP (SBP) $\geq 140 \mathrm{mmHg}$ or diastolic BP (DBP) $\geq 90 \mathrm{mmHg}$. Trained investigators assessed the blood pressure (BP) of the patients on at least three different occasions. All patients underwent a complete physical examination. Secondary causes of hypertension and heart, liver, and kidney diseases were excluded by patient history and by physical and laboratory examinations. After a 2-week, single-blind, placebo run-in period, all patients were treated orally with iptakalim (Thadweik Academy of Medicine, Beijing, China) at a single daily fixed dosage of $5 \mathrm{mg}$ (one tablet per day) for 4 consecutive weeks. Subsequently, patients whose BP was $<140 / 90 \mathrm{mmHg}$ continued the same dose regimen for another 4 weeks. In patients whose BP was not adequately controlled $(\geq 140 / 90 \mathrm{mmHg}$ ), the dose was doubled for the following 4 weeks. Patients were required to take their dose of iptakalim at approximately 9 a.m. and carefully record the time they took the tablet. Blood pressure was measured before taking the medicine on the first day and $24 \mathrm{~h}$ after 8 weeks of treatment. Three consecutive measurements were carried out on the right arm of quietly seated participants with a 1-min interval between replicates. When the difference between the measurements was $>4 \mathrm{mmHg}$, the patient was asked to rest for $5 \mathrm{~min}$, and then repeated measurements were carried out. In all of our analyses, an average of three consecutive blood pressure readings was used. Written informed consent was obtained from all patients enrolled in the study in accordance with principles of the Declaration of Helsinki. The protocol of the study was approved by the institutional Ethics Committee.

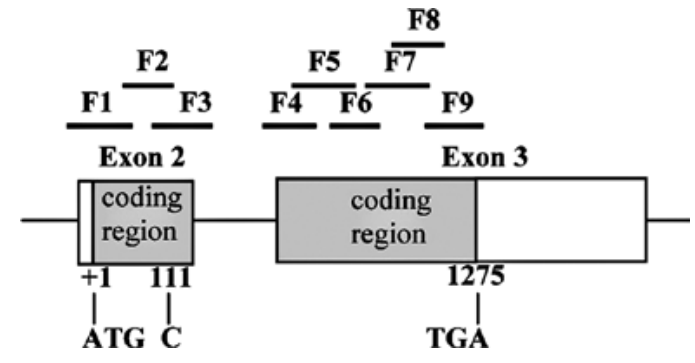

Figure 1. Structure of the coding region of the Kir6.1 gene. Nine PCR fragments are shown. The A of the ATG translation initiation site was used as nucleotide +1 . The average length of the PCR fragments used for SSCP was $\sim 260 \mathrm{bp}$.

DNA studies. Genomic DNA was extracted from peripheral blood leukocytes using standard procedures (Promega genomic DNA purification kit, USA). The Kir6.1 gene was amplified by PCR and screened using the PCR-SSCP method $(17,18)$. Primers of the Kir6.1 gene were designed using Primer3 (http://frodo.wi.mit.edu/primer3/) (Table I). The amplification was performed as follows: initial denaturation at $95^{\circ} \mathrm{C}$ for $5 \mathrm{~min}$ followed by 35 cycles of denaturation at $95^{\circ} \mathrm{C}$ for $30 \mathrm{sec}$, annealing at $58^{\circ} \mathrm{C}$ for $30 \mathrm{sec}$, and extension at $72^{\circ} \mathrm{C}$ for $1 \mathrm{~min}$, and final extension at $72^{\circ} \mathrm{C}$ for $5 \mathrm{~min}$. cDNA and protein sequences were numbered according to GenBank NM_004982 with nucleotide numbering beginning with the first Met (Fig. 1). The samples were heated at $100^{\circ} \mathrm{C}$ for $10 \mathrm{~min}$ and immediately chilled on ice. PCR products $(5 \mu \mathrm{l})$ were electrophoresed on a $8 \%$ non-denaturing acrylamide:bisacrylamide (29:1) gel in 1X TBE buffer. Gels were run overnight at a constant $5 \mathrm{~W}$ at $4^{\circ} \mathrm{C}$, and $\mathrm{SSCP}$ 
bands were visualized on the gels by silver staining. Direct sequencing (ABI 3730XL sequencer, Applied Biosystem) was used to confirm the results.

Statistical analysis. Values are expressed as the mean \pm SD. The means for continuous variables in the two groups were compared using the t-test. The prevalence of categorical variables was compared using the $\chi^{2}$ test. The blood pressure (BP) response was defined as the blood pressure before treatment minus the blood pressure at the end of the 8-week treatment. All tests were two-tailed, and $\mathrm{P}<0.05$ was considered statistically significant. The SPSS 13.0 statistical package (SPSS, Chicago, IL, USA) was used for analysis.

\section{Results}

PCR-SSCP and DNA sequencing were used to detect mutation of the Kir6.1 gene in 162 Chinese Han hypertensive patients treated by iptakalim. As shown in Fig. 2, single base pair substitution ( $\mathrm{C}$ to $\mathrm{T}$ ) at nucleotide position 111 in exon 2 of the coding region was detected in one female and three male patients. This substitution in four patients was heterozygous and did not result in an amino acid substitution (Ile37Ile, silent mutation). The I37I polymorphism was found to have a low prevalence in the Chinese Han hypertensive patients. The frequencies of the CC and CT genotypes were 97.5 and $2.5 \%$, respectively. The TT genotype was not found in this population. For the 162 patients in this study, there were no significant differences in age, gender, body mass index (BMI), baseline SBP and DBP between patients with the CC and patients with the $\mathrm{CT}$ genotype before iptakalim treatment (Table II). The BP of the four patients with the CT genotype was well controlled by iptakalim treatment. Comparison of the reductions in $\mathrm{BP}$ at 8 weeks post treatment in the patients with the I37I polymorphism showed that there was no significant difference in the SBP and DBP response between the patients with the CC and CT genotypes (Table II).
A

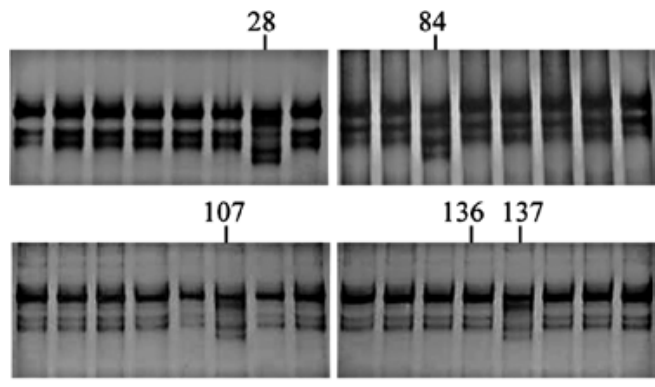

B

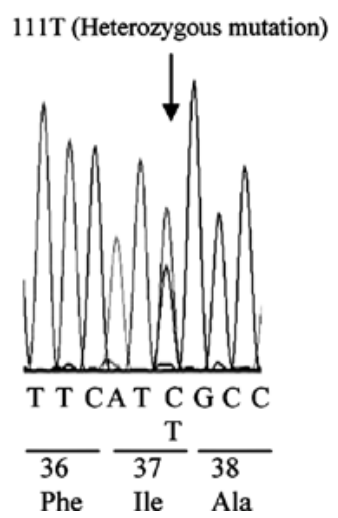

C

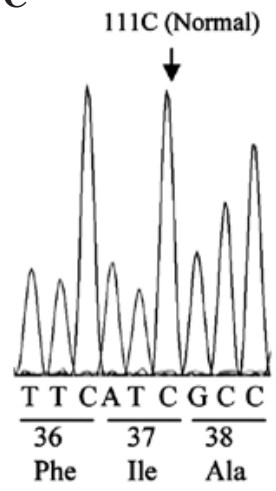

Figure 2. Representative electrophoretogram of PCR-SSCP for the F1 fragment in exon 2 (8\% polyacrylamide gel) (A) and the representive sequence pattern of the I37I polymorphism at nucleotide position 111 in exon 2 of the Kir6.1 gene (B and C). Four patients were found to exhibit the CT genotype (B, sample 28, 84, 107 and 137). The other 158 patients had the CC genotype (C, sample 136). Phe, phenylalanine; Ile, isoleucine and Ala, alanine.

\section{Discussion}

During screening of the entire coding region and partial screening of the intron of the Kir6.1 gene in 162 patients, a low prevalence of the I37I polymorphism in the exon 2 coding

Table II. Clinical characteristics of the patients and evaluation of the reduction in blood pressure (BP) at 8 weeks post treatment with iptakalim in patients with the I37I polymorphism.

\begin{tabular}{lccc}
\hline & \multicolumn{3}{c}{ Kir6.1 gene I37I genotype } \\
\cline { 2 - 4 } Characteristics & $\begin{array}{c}\mathrm{CC} \\
(\mathrm{n}=158)\end{array}$ & $\begin{array}{c}\mathrm{CT} \\
(\mathrm{n}=4)\end{array}$ & P-value $^{\mathrm{a}}$ \\
\hline Age, mean (SD), years & $55(9)$ & $59(12)$ & 0.395 \\
Gender (male/female) & $78 / 80$ & $1 / 3$ & 0.314 \\
Body mass index, mean (SD), kg/m² & $25.3(2.7)$ & $24.7(1.8)$ & 0.627 \\
Blood pressure, mean (SD), mmHg & & $149.3(16.6)$ & 0.587 \\
SBP & $152.7(12.6)$ & $99.7(4.2)$ & 0.876 \\
DBP & $100.0(4.1)$ & $12.9(24.4)$ & 0.787 \\
BP reduction, mean (SD), mmHg & $11.0(13.4)$ & $16.4(14.3)$ & 0.191 \\
$\Delta$ SBP & $11.0(7.8)$ & & \\
$\Delta$ DBP & & & \\
\hline
\end{tabular}

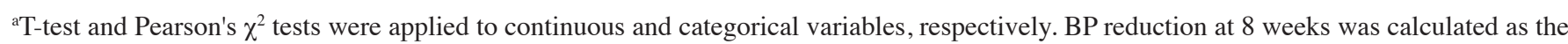
pretreatment BP minus post-treatment BP. SBP, systolic blood pressure; DBP, diastolic blood pressure. 
region was noted. Frequencies of the $\mathrm{CC}$ and $\mathrm{CT}$ genotypes were $158 / 162(97.5 \%)$ and $4 / 162(2.5 \%)$, respectively. The TT genotype was not found in this population. There were no significant differences in BP response to iptakalim between the patients with the CC genotype and those with the CT genotype. No other mutations or polymorphisms were detected in the remaining patients. The results indicate that abnormality in the primary structure of Kir6.1 may not be involved in the genetic pathogenesis of essential hypertension and the BP response to iptakalim in Chinese Han hypertensive patients.

The I37I mutation was previously detected in one female Japanese patient with coronary spastic angina (CSA). No other mutation was detected in the genetic analysis of the Kir6.1 gene in 19 Japanese patients with CSA (19). A complete mutational analysis of the Kir6.1 gene was performed in a series of 18 Italian patients with impaired coronary vasomotility, and no mutations were detected in the sample analyzed, thus suggesting that Kir6.1 gene aberrations are not a common cause of abnormal coronary vasomotility in Italians (20). A rare missense variant in exon 3 of the Kir6.1 gene was found to be associated with ventricular fibrillation with prominent early repolarization (21). The mutational analysis of the Kir6.1 gene among various ethnic groups showed that it may be so important that no mutations or polymorphisms that alter the primary structure of Kir6.1 are allowed. This is in agreement with the result of a Kir6.1 gene knockout experiment which found that deficiency of the Kir6.1 $\mathrm{K}_{\text {ATP }}$ channel imparts fatal susceptibility to endotoxemia (22).

To our knowledge, this study is the first to investigate polymorphisms of the Kir6.1 gene and the association of the I37I polymorphism with $\mathrm{BP}$ and $\mathrm{BP}$ response to antihypertensive drugs in a Chinese Han hypertensive population. One limitation of our study is that, even though our sample size (162 patients) was larger than previous Kir6.1 gene mutation studies (19-21), our study population may be too small to confirm an association of the I37I polymorphism and BP response to iptakalim due to the low prevalence of this polymorphism in our study. Therefore a larger sample is needed to more accurately determine whether or not the I37I polymorphism is associated with $\mathrm{BP}$ response to iptakalim treatment. Iptakalim preferentially activates the SUR2B/Kir6.1 subtype of $\mathrm{K}_{\text {ATP }}$ channels $(13,23)$, and the SUR2B gene may be an important candidate gene for pharmacogenetics research of iptakalim.

In conclusion, we demonstrated that abnormality in the primary structure of Kir6.1 is not involved in the genetic pathogenesis of essential hypertension in Chinese Han hypertensive patients and the I37I polymorphism may have no effect on the BP response to iptakalim treatment.

\section{Acknowledgements}

This study was supported by grants from the National Key Technologies R\&D Program of China (no. 2008ZXJ09004018). We thank the patients for their participation.

\section{References}

1. Zhang S, Mao G, Zhang Y, et al: Association between human atrial natriuretic peptide Val7Met polymorphism and baseline blood pressure, plasma trough irbesartan concentrations, and the antihypertensive efficacy of irbesartan in rural Chinese patients with essential hypertension. Clin Ther 27: 1774-1784, 2005.
2. Trotta R, Donati MB and Iacoviello L: Trends in pharmacogenomics of drugs acting on hypertension. Pharmacol Res 49: 351-356, 2004.

3. Arnett DK and Claas SA: Pharmacogenetics of antihypertensive treatment: detailing disciplinary dissonance. Pharmacogenomics 10: 1295-1307, 2009.

4. Sherva R, Ford CE, Eckfeldt JH, Davis BR, Boerwinkle E and Arnett DK: Pharmacogenetic effect of the stromelysin (MMP3) polymorphism on stroke risk in relation to antihypertensive treatment: the genetics of hypertension associated treatment study. Stroke 42: 330-335, 2011.

5. Duarte JD, Lobmeyer MT, Wang Z, et al: Lack of association between polymorphisms in STK39, a putative thiazide response gene, and blood pressure response to hydrochlorothiazide. Pharmacogenet Genomics 20: 516-519, 2010.

6. Suonsyrjä T, Donner K, Hannila-Handelberg T, Fodstad H, Kontula K and Hiltunen TP: Common genetic variation of beta1- and beta2-adrenergic receptor and response to four classes of antihypertensive treatment. Pharmacogenet Genomics 20: 342-345, 2010

7. Nichols CG: KATP channels as molecular sensors of cellular metabolism. Nature 440: 470-476, 2006.

8. Wang $\mathrm{H}$, Zhang YL and Chen YP: Targeting small arteries of hypertensive status with novel ATP-sensitive potassium channel openers. Curr Vasc Pharmacol 3: 119-124, 2005.

9. Wang H, Long CL and Zhang YL: A new ATP-sensitive potassium channel opener reduces blood pressure and reverses cardiovascular remodeling in experimental hypertension. J Pharmacol Exp Ther 312: 1326-1333, 2005.

10. Gao S, Long $\mathrm{CL}$, Wang $\mathrm{RH}$ and Wang $\mathrm{H}$ : $\mathrm{K}_{\mathrm{ATP}}$ activation prevents progression of cardiac hypertrophy to failure induced by pressure overload via protecting endothelial function. Cardiovasc Res 83: 444-456, 2009.

11. Wang $\mathrm{H}$, Tang $\mathrm{Y}$, Wang $\mathrm{L}$, Long $\mathrm{CL}$ and Zhang YL: ATP-sensitive potassium channel openers and 2,3-dimethyl2-butylamine derivatives. Curr Med Chem 14: 133-155, 2007.

12. Chen YP, Qiu CR and Wang H: Cardiovascular pharmacological characterization of novel 2,3-dimethyl-2-butylamine derivatives in rats. Life Sci 75: 2131-2142, 2004.

13. Chen YP, Cui WY and Wang H: Selective actions of iptakalim on the subtypes of $\mathrm{K}_{\text {ATP }}$ channels. Chin Pharmacol Bull 22: 278-284, 2006

14. Long CL, Qin XC, Pan ZY, et al: Activation of ATP-sensitive potassium channels protects vascular endothelial cells from hypertension and renal injury induced by hyperuricemia. J Hypertens 26: 2326-2338, 2008.

15. Duan RF, Cui WY, Gao HY, et al: Correlation between ACE gene I/D polymorphism and clinical efficacy of iptakalim in Chinese Han hypertensive population. Chin J Clin Pharmacol Ther 15: 961-966, 2010.

16. Evans WE and Relling MV: Pharmacogenomics: translating functional genomics into rational therapeutics. Science 286: 487-491, 1999.

17. Bettinaglio P, Galbusera A, Caprioli J, et al: Single strand conformation polymorphism (SSCP) as a quick and reliable method to genotype M235T polymorphism of angiotensinogen gene. Clin Biochem 35: 363-368, 2002.

18. Jun Lu, Shan Jiang, Song Ye and Chaopin Li: Detecting drug resistant genetic mutation among pneumoconiosis patients complicated with tuberculosis in Mycobacterium tuberculosis L-forms application of PCR-SSCP technique in Huainan mining district. J Nanjing Med Univ 3: 180-184, 2007.

19. Tomita H, Sasaki S, Osanai T, et al: Mutational analysis of Kir6.1 in Japanese patients with coronary spastic angina. Int J Mol Med 18: 589-591, 2006.

20. Emanuele E, Falcone C, Carabela M, et al: Absence of Kir6.1/ KCNJ8 mutations in Italian patients with abnormal coronary vasomotion. Int J Mol Med 12: 509-512, 2003.

21. Haïssaguerre M, Chatel S, Sacher F, et al: Ventricular fibrillation with prominent early repolarization associated with a rare variant of KCNJ8/KATP channel. J Cardiovasc Electrophysiol 20: 93-98, 2009.

22. Kane GC, Lam CF, O'Cochlain F, et al: Gene knockout of the KCNJ8-encoded Kir6.1 K(ATP) channel imparts fatal susceptibility to endotoxemia. FASEB J 20: 2271-2280, 2006.

23. Pan Z, Huang J, Cui W, Long C, Zhang Y and Wang H: Targeting hypertension with a new adenosine triphosphate-sensitive potassium channel opener iptakalim. J Cardiovasc Pharmacol 56: $215-228,2010$ 